\title{
ON THE QUASI-STATIONARY DISTRIBUTION FOR QUEUEING NETWORKS WITH DEFECTIVE ROUTING
}

\author{
RICHARD J. BOUCHERIE ${ }^{1}$
}

(Received 10 April 1995; revised 6 August 1995)

\begin{abstract}
This note introduces quasi-local-balance for discrete-time Markov chains with absorbing states. From quasi-local-balance product-form quasi-stationary distributions are derived by analogy with product-form stationary distributions for Markov chains that satisfy local balance.
\end{abstract}

\section{Introduction}

Product-form distributions have proven to be a useful tool in the analysis of queueing networks in equilibrium. Based on product-form distributions various tools for the analysis of performance measures have been derived. In applications it is often found that also for systems that do not approach equilibrium, results derived from productform distributions give adequate approximations of the system. This note provides an explanation of the robustness of queueing-network formulas. It will be shown that the quasi-stationary distribution for queueing networks with defective routing is of product-form. This result allows one to use known techniques for queueing networks in equilibrium in the quasi-stationary regime.

This note considers finite Markov chains with a single communicating class of transient states at which the behaviour of the Markov chain is of interest. As absorption into the remaining states is certain, the equilibrium distribution at the transient communicating class is of little value for the analysis of the process. However, it is often of interest to calculate the limiting distribution of the Markov chain being in a transient state before absorption takes place. When the time to absorption is sufficiently long, under some conditions, this distribution is known as the quasi-stationary distribution. The theory for quasi-stationary distributions of Markov chains with finite

\footnotetext{
${ }^{1}$ Universiteit van Amsterdam, Department of Econometrics, Roetersstraat 11, 1018 WB Amsterdam, The Netherlands
}

(C) Australian Mathematical Society, 1997, Serial-fee code 0334-2700/97 
state space is well-developed. The product-form results of this note can be seen as examples of the results in the papers of Darroch and Seneta [2], [3]. Further results on quasi-stationary distributions and related notions such as $R$-recurrence can, for example, be found in Vere-Jones [8], Seneta and Vere-Jones [7], Kesten [5].

The results of this note are mainly designed to analyse queueing networks of the Jackson type. To obtain a more comprehensive formulation of the results, the theory is formulated for queueing networks with batch routing. Section 2 presents the results for discrete-time Markov chains. In Section 3 the results are illustrated through the jump chain of a Jackson network. Furthermore, the results are extended to continuous-time Markov chains.

\section{Discrete-time models}

Consider a queueing network consisting of $N$ queues or stations. Assume that the queueing network can be represented by a discrete-time Markov chain $\boldsymbol{X}=$ $\left\{X_{k}, k=0,1,2, \ldots\right\}$ with finite state space $S$ that describes the number of customers at the stations of the network; $\boldsymbol{X}$ makes a transition when customers move among the queues. A state $n=\left(n_{1}, \ldots, n_{N}\right) \in S$ is a vector with components $n_{i} \in \mathbb{N}_{0}$ denoting the number of customers at queue $i, i=1, \ldots, N$.

For $\mathbf{0} \leq \boldsymbol{g} \leq \boldsymbol{n}$ (componentwise) let $\boldsymbol{\mu}(\boldsymbol{g}, \boldsymbol{n})$. denote the probability that a batch $\boldsymbol{g}=\left(g_{1}, \ldots, g_{N}\right)$, containing $g_{i}$ customers at station $i, i=1, \ldots, N$, leaves the stations when the network is in state $n$. In accordance with the literature on productform distributions ( $c f$. Boucherie and van Dijk [1], Henderson and Taylor [4]), assume that $\psi: \mathbb{N}_{0}^{N} \rightarrow[0, \infty), \phi: \mathbb{N}_{0}^{N} \rightarrow(0, \infty), \theta: \mathbb{N}_{0}^{N} \rightarrow[0, \infty)$, exist such that

$$
\mu(\boldsymbol{g}, \boldsymbol{n})=\frac{\psi(\boldsymbol{n}-\boldsymbol{g}) \theta(\boldsymbol{g})}{\phi(\boldsymbol{n})}, \quad \sum_{\mathbf{0} \leq \boldsymbol{g} \leq \boldsymbol{n}} \frac{\psi(\boldsymbol{n}-\boldsymbol{g}) \theta(\boldsymbol{g})}{\phi(\boldsymbol{n})}=1 \quad \text { for all } \boldsymbol{n} \in S .
$$

Upon departure from the queues a batch $g$ becomes a batch $\boldsymbol{g}^{\prime}=\left(g_{1}^{\prime}, \ldots, g_{N}^{\prime}\right)$ of arrivals, $g_{i}^{\prime}$ customers arriving at station $i$, with probability $p\left(g, g^{\prime}\right)$. The transition probabilities of $\boldsymbol{X}$ are

$$
q\left(\boldsymbol{n}, \boldsymbol{n}^{\prime}\right)=\sum_{\left\{m, \boldsymbol{g}, \boldsymbol{g}^{\prime}: m+\boldsymbol{g}=n, m+g^{\prime}=n^{\prime}\right\}} q\left(\boldsymbol{g}, \boldsymbol{g}^{\prime} ; \boldsymbol{m}\right), \quad n, \boldsymbol{n}^{\prime} \in S,
$$

where

$$
q\left(\boldsymbol{g}, \boldsymbol{g}^{\prime} ; \boldsymbol{m}\right)=\frac{\psi(\boldsymbol{m}) \theta(\boldsymbol{g})}{\phi(\boldsymbol{m}+\boldsymbol{g})} p\left(\boldsymbol{g}, \boldsymbol{g}^{\prime}\right), \quad \boldsymbol{g}, \boldsymbol{g}^{\prime}, \boldsymbol{m} \in \mathbb{N}_{0}^{N}
$$

We are interested in the case in which $\boldsymbol{X}$ has an absorbing set $S^{\prime} \subset S ; X$ terminates upon arrival in $S^{\prime} \subset S$. For this process $X$, let $T\left(n_{0}\right)$ be the time until absorption 
conditional on $X$ starting in $n_{0} \in S$. The quasi-stationary distribution $\pi=(\pi(n), n \in$ $S \backslash S^{\prime}$ ) is defined by

$$
\pi(n)=\lim _{t \rightarrow \infty} P\left(X_{t}=n \mid X_{0}=n_{0}, T\left(n_{0}\right)>t\right),
$$

if the limit exists and is independent of the initial state $\boldsymbol{n}_{0}$. The quasi-stationary distribution corresponds to the probability of being in state $\boldsymbol{n}$ given that absorption has not yet occurred. The limit then accounts for those sample paths for which the time to absorption is infinite, which supports the independence of $\boldsymbol{n}_{0}$ for $\pi$.

For discrete-time Markov chains with finite state space, under the assumption that the matrix

$$
Q:=\left(q\left(\boldsymbol{n}, \boldsymbol{n}^{\prime}\right), \boldsymbol{n}, \boldsymbol{n}^{\prime} \in S \backslash S^{\prime}\right)
$$

is primitive, Darroch and Seneta [2] show that the quasi-stationary distribution can be obtained as the unique Perron-Frobenius left eigenvector of $Q$, that is, with $\alpha<1$ the maximal real eigenvalue of $Q$ ( $\alpha<1$ because $Q$ is substochastic), it must be that $\pi$ is the unique solution of

$$
\pi Q=\alpha \pi
$$

Below we will show that the quasi-stationary distribution $\pi$ is of product-form when the reduced state space $S \backslash S^{\prime}$ is of a form that would be compatible with a productform equilibrium distribution in a way to be made precise below. The underlying idea is the notion of quasi-local-balance that replaces the notion of local balance used to obtain product-form equilibrium distributions. The notion of quasi-local-balance is related to the routing probabilities $p\left(g, g^{\prime}\right)$. Let us therefore first describe these routing probabilities in more detail.

AsSUMPTION 2.1. Let $G \subset \mathbb{N}_{0}^{N}$ be such that $\theta(g)>0$ if and only if $g \in G$. Assume that $P=\left(p\left(g, g^{\prime}\right), g, g^{\prime} \in G\right)$ is substochastic and primitive. Let $\alpha^{\prime}$ be the maximal real eigenvalue of $P$ and $c>0$ the corresponding left eigenvector:

$$
c P=\alpha^{\prime} c .
$$

The set $G$ defined above is the set of all batches that can be served. Hence, the routing matrix $P$ contains entries for batches that can only be served. The substochasticity of $P$ implies that some of the batches $g \in G$ can route to batches $g^{\prime} \notin G$, batches that cannot be served.

By analogy with local balance for ergodic Markov chains, we now define quasilocal-balance for Markov chains with an absorbing set. Quasi-local-balance can be seen as the extension of (2.5) to the state space level. 
DEFINITION 2.2. The Markov chain X satisfies quasi-local-balance for some $\alpha$ when for all $\boldsymbol{m}, \boldsymbol{g}$ such that $\boldsymbol{m}+\boldsymbol{g} \in S \backslash S^{\prime}, \boldsymbol{g} \in G$, a positive solution $\pi=\left(\pi(\boldsymbol{n}), \boldsymbol{n} \in S \backslash S^{\prime}\right)$ exists of

$$
\sum_{g^{\prime} \in G} \pi\left(m+g^{\prime}\right) q\left(g^{\prime}, g ; m\right)=\alpha \pi(m+g) \mu(g, m+g) .
$$

We also say that $\pi$ satisfies quasi-local-balance for $\alpha$ at $S \backslash S^{\prime}$.

The relation between quasi-local-balance and (2.4) is similar to the relation between local balance and global balance.

LEMMA 2.3. If $\pi$ satisfies quasi-local-balance for $\alpha$ at $S \backslash S^{\prime}$ then $\alpha$ is the PerronFrobenius eigenvalue of $Q, \pi$ satisfies (2.4), and $\pi$ is the unique quasi-stationary distribution of $\dot{X}$.

ProOF. Summing the left-hand side of (2.6) over $\left\{g, m: m+g=n, n \in S \backslash S^{\prime}\right\}$ gives that

$$
\begin{aligned}
& \sum_{\{g, m: m+g=n\}} \sum_{g^{\prime} \in G} \pi\left(\boldsymbol{m}+\boldsymbol{g}^{\prime}\right) q\left(\boldsymbol{g}^{\prime}, \boldsymbol{g} ; \boldsymbol{m}\right) \\
& \quad=\sum_{\{g, m: m+g=n\}} \sum_{\left\{g^{\prime} \in G: m+g^{\prime} \in S \backslash S^{\prime}\right\}} \pi\left(\boldsymbol{m}+\boldsymbol{g}^{\prime}\right) q\left(\boldsymbol{g}^{\prime}, \boldsymbol{g} ; \boldsymbol{m}\right) \\
& \quad=\sum_{n^{\prime} \in S \backslash S^{\prime}} \sum_{\left(\boldsymbol{m}, \boldsymbol{g}^{\prime} \cdot \boldsymbol{g}: \boldsymbol{m}+g^{\prime}=n^{\prime}, \boldsymbol{m}+\boldsymbol{g}=n\right\}} \pi\left(\boldsymbol{m}+\boldsymbol{g}^{\prime}\right) q\left(\boldsymbol{g}^{\prime}, \boldsymbol{g} ; \boldsymbol{m}\right) \\
& \quad=\sum_{n^{\prime}} \pi\left(\boldsymbol{n}^{\prime}\right) q\left(\boldsymbol{n}^{\prime}, \boldsymbol{n}\right) .
\end{aligned}
$$

We have used that $\pi\left(\boldsymbol{m}+\boldsymbol{g}^{\prime}\right) q\left(\boldsymbol{g}^{\prime}, \boldsymbol{g} ; \boldsymbol{m}\right)=0$ for $\boldsymbol{m}+\boldsymbol{g}^{\prime} \notin S \backslash S^{\prime}, \boldsymbol{m}+\boldsymbol{g} \in S \backslash S$ in the first equality. The second equality is due to the primitiveness of $P$, and the last equality is obtained from (2.2).

Summing the right hand side over $\{\boldsymbol{g}, \boldsymbol{m}: \boldsymbol{m}+\boldsymbol{g}=\boldsymbol{n}\}$, using (2.1), gives that

$$
\sum_{\{g, m: m+g=n\}} \alpha \pi(m+g) \mu(g, m+g)=\alpha \pi(n),
$$

which establishes that $\pi$ satisfies (2.4).

We have found a positive number $\alpha$ and a vector $\pi \geq 0, \pi \neq 0$, such that $\pi Q=\alpha \pi$. Theorem 1.6, p. 23, of Seneta [6] implies that $\alpha$ is the Perron-Frobenius eigenvalue of $Q$, which also implies that $\pi$ is the unique Perron-Frobenius left eigenvector of $Q$.

We are now ready to state the main result of this section. 
THEOREM 2.4. Assume that $H: S \backslash S^{\prime} \rightarrow(0, \infty)$ exists such that for all $g, g^{\prime} \in G$, $\boldsymbol{m}+\boldsymbol{g}, \boldsymbol{m}+\boldsymbol{g}^{\prime} \in S \backslash S^{\prime}$

$$
\frac{H\left(m+g^{\prime}\right) \theta\left(g^{\prime}\right)}{H(m+g) \theta(g)}=\frac{c\left(g^{\prime}\right)}{c(g)}
$$

Then $\pi$ given by

$$
\pi(\boldsymbol{n})=B \phi(n) H(n), \quad n \in S \backslash S^{\prime}
$$

satisfies quasi-local-balance for $\alpha=\alpha^{\prime}$, and $\pi$ is the unique quasi-stationary distribution of $\boldsymbol{X}$ at $S \backslash S^{\prime}$ (B is the normalising constant).

PROOF. It is sufficient to show that $\pi$ satisfies quasi-local-balance (2.6) at $S \backslash S^{\prime}$ with $\alpha=\alpha^{\prime}$. For $\boldsymbol{m}, \boldsymbol{g}$ such that $\boldsymbol{m}+\boldsymbol{g} \in S \backslash S^{\prime}$ :

$$
\begin{aligned}
\sum_{\boldsymbol{g}^{\prime} \in G} \frac{\pi\left(\boldsymbol{m}+\boldsymbol{g}^{\prime}\right) q\left(\boldsymbol{g}^{\prime}, \boldsymbol{g} ; \boldsymbol{m}\right)}{\pi(\boldsymbol{m}+\boldsymbol{g})} & =\sum_{\boldsymbol{g}^{\prime} \in G} \frac{\psi(\boldsymbol{m}) \theta(\boldsymbol{g})}{\phi(\boldsymbol{m}+\boldsymbol{g})} \frac{H\left(\boldsymbol{m}+\boldsymbol{g}^{\prime}\right) \theta\left(\boldsymbol{g}^{\prime}\right)}{H(\boldsymbol{m}+\boldsymbol{g}) \theta(\boldsymbol{g})} p\left(\boldsymbol{g}^{\prime}, \boldsymbol{g}\right) \\
& \stackrel{(2.7)}{=} \frac{\psi(\boldsymbol{m}) \theta(\boldsymbol{g})}{\phi(\boldsymbol{m}+\boldsymbol{g})} \sum_{\boldsymbol{g}^{\prime}} \frac{c\left(\boldsymbol{g}^{\prime}\right)}{c(\boldsymbol{g})} p\left(\boldsymbol{g}^{\prime}, \boldsymbol{g}\right) \\
& \stackrel{(2.5)}{=} \alpha^{\prime} \frac{\psi(\boldsymbol{m}) \theta(\boldsymbol{g})}{\phi(\boldsymbol{m}+\boldsymbol{g})} \\
& \stackrel{(2.1)}{=} \alpha^{\prime} \mu(\boldsymbol{g}, \boldsymbol{m}+\boldsymbol{g}) .
\end{aligned}
$$

REMARK (Local balance). Quasi-local-balance determines the state space $S \backslash S^{\prime}$ for which the equations (2.5) at $G$ can be translated to a set of equations at the state space. Similar to local balance for product-form results for the equilibrium distribution of ergodic Markov chains, quasi-local-balance (2.6) restricts the reduced state space $S \backslash S^{\prime}$ considerably. In fact, due to quasi-local-balance, the reduced state space $S \backslash S^{\prime}$ must be such that a Markov chain with stochastic routing matrix at $G$ could have a product-form equilibrium distribution. If $\boldsymbol{g} \in G, \boldsymbol{m}+\boldsymbol{g} \in S \backslash S^{\prime}$, and $p\left(\boldsymbol{g}, \boldsymbol{g}^{\prime}\right)>0$ for some $g^{\prime} \in G$, then $\boldsymbol{m}+\boldsymbol{g}^{\prime} \in S \backslash S^{\prime}$ for all $\boldsymbol{g}^{\prime} \in G$, a direct consequence of the primitiveness of $P$.

REMARK (Absorption). Termination of $\boldsymbol{X}$ occurs due to the substochasticity of the routing matrix $P$. $X$ terminates when a batch $g$ is routed to a batch $g^{\prime} \notin G$. The probability that $X$ terminates from state $n \in S \backslash S^{\prime}$ is

$$
q\left(\boldsymbol{n}, S^{\prime}\right)=\sum_{\boldsymbol{g} \in \boldsymbol{G}} \mu(\boldsymbol{g}, \boldsymbol{n})\left(\mathbf{1}-\sum_{\boldsymbol{g}^{\prime} \in G} p\left(\boldsymbol{g}, \boldsymbol{g}^{\prime}\right)\right), \quad \boldsymbol{n} \in S \backslash S^{\prime} .
$$

As $S^{\prime}$ is an absorbing set, the transition rates (2.2) are such that $q\left(n, n^{\prime}\right)=0$ for $n \in S^{\prime}, n^{\prime} \in S \backslash S^{\prime}$, which imposes restrictions on the rates $q\left(\boldsymbol{g}, \boldsymbol{g}^{\prime} ; \boldsymbol{m}\right)$. These rates do not influence the quasi-stationary distribution at $S \backslash S^{\prime}$, and the functional form of $q\left(g, g^{\prime} ; m\right)$ as given in (2.3) is not required when $m+g \in S^{\prime}$. 
REMARK (Decay parameter). The Perron-Frobenius eigenvalue $\alpha$ is the decay parameter or convergence norm of the substochastic matrix $Q$ and can also be found from

$$
1 / \alpha=\sup _{z}\left\{\sum_{n=0}^{\infty} Q^{k}\left(\boldsymbol{n}, \boldsymbol{n}^{\prime}\right) z^{k}<\infty\right\} .
$$

A result of Theorem 2.4 is that the decay parameter of $P$ equals the decay parameter of $Q$. This is not surprising, since $\boldsymbol{X}$ terminates due to the substochasticity of $P$.

REMARK (Countable state spaces). The results of this note are presented for Markov chains with a finite state space only. In this case, the left Perron-Frobenius eigenvector determines the quasi-stationary distribution. For countable state spaces $S \backslash S^{\prime}$ we cannot draw this conclusion. The measure $\pi$ found in Theorem 2.4 is still a left eigenvector for the eigenvalue $\alpha$. To conclude that $\pi$ is indeed the quasi-stationary distribution it is sufficient to establish that the transition matrix $Q$ is $R$-positive, and that $\pi$ is summable (Seneta and Vere-Jones [7]). Also when the $Q$ matrix is not $R$-positive, for Markov chains with bounded jumps Kesten [5] shows, under some conditions, that we can conclude that a positive left eigenvector of $Q$ with eigenvalue $1 / R$, is indeed the quasi-stationary distribution. The eigenvalue $\alpha$ obtained in Theorem 2.4 is not guaranteed to coincide with $1 / R$. Moreover, the states of a queueing network cannot be ordered such that jumps are bounded. Therefore we cannot conclude that the measure $\pi$ found in Theorem 2.4 is the quasi-stationary distribution unless $S \backslash S^{\prime}$ is finite.

\section{Examples and extensions}

In this section we will present some examples of discrete-time queueing networks for which the quasi-stationary distribution has a product-form. In the first example we consider the jump-chain of a Jackson network. The second example uses the results for Jackson networks to obtain the quasi-stationary distribution for a discrete-time queueing network with independently routing customers. Finally, we present the extension of Theorem 2.4 to continuous-time Markov chains.

3.1. A discrete-time Jackson network We consider a closed Jackson network consisting of $N$ stations in which customers route singly. The process can be seen as a model for a manufacturing system, stations representing machines, and customers representing produced items. The system might fail due to defective routing, that is an item transported from one machine to another might be damaged. When this occurs the manufacturing system is stopped to remove the damaged item. Below we will show that the quasi-stationary distribution for this manufacturing system has a product-form. 
The jump chain of the Jackson network records the number of customers at the stations and ignores the sojourn time in the states. This jump chain is a discrete-time model and fits the requirements of Theorem 2.4. The service probabilities are

$$
\mu\left(\boldsymbol{e}_{i}, \boldsymbol{n}\right)=\frac{\psi\left(\boldsymbol{n}-\boldsymbol{e}_{i}\right) \theta\left(\boldsymbol{e}_{i}\right)}{\phi(\boldsymbol{n})}, \quad i=1, \ldots, N
$$

where $\boldsymbol{e}_{i}$ denotes the $i$-th unit vector, and $\mu\left(\boldsymbol{e}_{i}, \boldsymbol{n}\right)$ satisfies (2.1). Upon completion at station $i$ a customer is routed to station $j$ with probability $p_{i j}$. Assume that $P=\left(p_{i j}, i, j=1, \ldots, N\right)$ is primitive. While routing a customer is damaged with probability $1-\sum_{j=1}^{N} p_{i j}, i=1, \ldots, N$. The probability that the network terminates from state $n$ is therefore given in (2.8).

Let $\alpha^{\prime}$ be the Perron-Frobenius eigenvalue of $P$, and $c=\left(c_{1}, \ldots, c_{N}\right)$ the PerronFrobenius eigenvector. From Theorem 2.4 we then obtain that the quasi-stationary distribution of the network that starts with $M$ identical customers is given by

$$
\pi(\boldsymbol{n})=\boldsymbol{B} \phi(\boldsymbol{n}) \prod_{k=1}^{N}\left(c_{k} \theta\left(\boldsymbol{e}_{k}\right)\right)^{n_{k}}, \quad \boldsymbol{n} \in\left\{\boldsymbol{n}: \sum_{i=1}^{N} n_{i}=M\right\},
$$

where $B$ is the normalising constant that can be determined using standard techniques known for product-form queueing networks. The decay parameter is $\alpha=\alpha^{\prime}$.

REMARK (Primitiveness of $P$ ). The assumption that $P$ is primitive restricts the queueing networks under consideration. For example, a tandem line with absorbing last queue does not have a primitive routing matrix. On the other hand, in the present setting the assumption of primitiveness is quite natural, since we are interested only in those networks for which sample paths of unbounded length are possible. This is clearly not the case for the tandem network.

REMARK (Product-form). This example illustrates the power of product-form distributions for the analysis of manufacturing systems. In practical situations the assumption that the system is in equilibrium is usually not satisfied. Still, in many applications, it is observed that product-form distributions do give adequate results. The results of this paper provide an explanation of the robustness of queueing network formulas also in situations where the obvious assumptions are not satisfied: the form of the distribution in the quasi-stationary regime is the same as the form of the distribution in equilibrium. Therefore, when the parameters figuring in the distribution are obtained from observations of the application, fitting a product-form distribution is justified.

\subsection{Discrete-time queueing networks with independently routing customers}

Consider a discrete-time closed queueing network consisting of $N$ stations. Assume 
that the service probabilities satisfy (2.1), and assume that customers route independently according to the routing probabilities $p_{i j}$ defined above. The probability that a batch $g \in G$ is routed to a batch $g^{\prime} \in G$ is then given by ( $c f$. Boucherie and van Dijk [1])

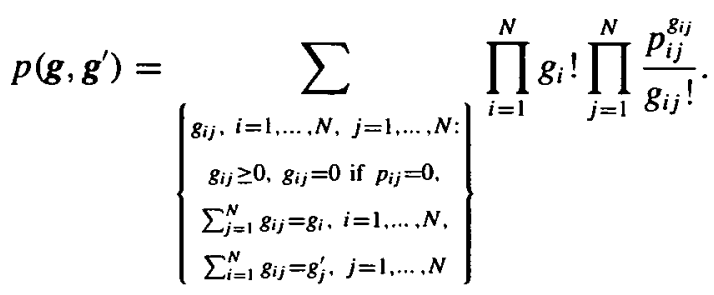

The substochasticity of $\left(p_{i j}, i, j=1, \ldots, N\right)$ implies that $P=\left(p\left(g, g^{\prime}\right), g, g^{\prime} \in G\right)$ is substochastic too. The assumption that $P$ is primitive implies that there is a constant $M$ such that $G=\left\{g: \sum_{j=1}^{N} g_{j}=M\right\}$. This implies that the size of the batch of customers that can be served is fixed (see Assumption 2.1).

Consider a discrete-time network containing $K \geq M$ customers. The network terminates as soon as a batch $g$ is routed to a batch $g^{\prime}$ with $\sum_{i=1}^{N}\left(g_{i}^{\prime}-g_{i}\right) \neq 0$, that is, as soon as a batch is damaged while routing. It can easily be shown that

$$
c(\boldsymbol{g})=\prod_{k=1}^{N} \frac{c_{i}^{g_{i}}}{g_{i} !}, \quad g \in G,
$$

is the Perron-Frobenius eigenvector, with corresponding eigenvalue $\left(\alpha^{\prime}\right)^{M}$ of $P$. For $\theta(g)=\left[\prod_{k=1}^{N} g_{k} !\right]^{-1}$, the quasi-stationary distribution is given in (3.1).

3.3. Continuous-time Markov chains For continuous-time Markov chains the quasi-stationary distribution can be obtained by analogy with the result of Theorem 2.4. To this end, consider a continuous-time Markov chain with transition rates

$$
\begin{aligned}
q\left(\boldsymbol{n}, \boldsymbol{n}^{\prime}\right) & =\sum_{\left\{m, \boldsymbol{g} \cdot \boldsymbol{g}^{\prime}: m+g=n, m+g^{\prime}=n^{\prime}\right\}} q\left(\boldsymbol{g}, \boldsymbol{g}^{\prime} ; \boldsymbol{m}\right), \quad \boldsymbol{n}^{\prime} \neq \boldsymbol{n}, \boldsymbol{n}, \boldsymbol{n}^{\prime} \in S, \\
q(\boldsymbol{n}, \boldsymbol{n}) & =-\sum_{\boldsymbol{n}^{\prime} \in S, \boldsymbol{n}^{\prime} \neq \boldsymbol{n}} q\left(\boldsymbol{n}, \boldsymbol{n}^{\prime}\right), \quad n \in S,
\end{aligned}
$$

where

$$
q\left(\boldsymbol{g}, \boldsymbol{g}^{\prime} ; \boldsymbol{m}\right)=\mu(\boldsymbol{g}, \boldsymbol{n}) p\left(\boldsymbol{g}, \boldsymbol{g}^{\prime}\right), \quad \boldsymbol{g}, \boldsymbol{g}^{\prime}, \boldsymbol{m} \in \mathbb{N}_{0}^{N} .
$$

Assume that $\mu(g, n)$ satisfies (2.1), and that Assumption 2.1 is satisfied. Let $S^{\prime}$ be the absorbing set. The transition rate from $n \in S \backslash S^{\prime}$ to $S^{\prime}$ is given in (2.8). We have the following result. 
THEOREM 3.1. Assume that $H: S \backslash S^{\prime} \rightarrow(0, \infty)$ exists such that for all $g, g^{\prime} \in G$, $\boldsymbol{m}+\boldsymbol{g}, \boldsymbol{m}+\boldsymbol{g}^{\prime} \in S \backslash S^{\prime}$

$$
\frac{H\left(m+g^{\prime}\right) \theta\left(g^{\prime}\right)}{H(m+g) \theta(g)}=\frac{c\left(g^{\prime}\right)}{c(g)}
$$

Then $\pi$ given by

$$
\pi(\boldsymbol{n})=B \phi(n) H(n), \quad n \in S \backslash S^{\prime}
$$

is the unique quasi-stationary distribution of $X$ at $S \backslash S^{\prime}$ ( $B$ is the normalising constant). The decay parameter is $\alpha=\alpha^{\prime}-1$.

PROOF. Darroch and Seneta [1967] show that it is sufficient to prove that $\pi$ is the unique Perron-Frobenius eigenvector with Perron-Frobenius eigenvalue $\alpha$ for the matrix $Q=\left(q\left(\boldsymbol{n}, \boldsymbol{n}^{\prime}\right), \boldsymbol{n}, \boldsymbol{n}^{\prime} \in S \backslash S^{\prime}\right)$. Insertion of the definition of the transition rates in the equations $\pi Q=\alpha \pi$ gives that $\pi$ and $\alpha$ must be determined from

$$
\sum_{\left\{n^{\prime} \in S \backslash S^{\prime}, n^{\prime} \neq n\right\}}\left\{\pi\left(n^{\prime}\right) q\left(n^{\prime}, n\right)-\pi(n) q\left(n, n^{\prime}\right)\right\}=\alpha \pi(n)+\sum_{n^{\prime} \in S^{\prime}} \pi(n) q\left(n, n^{\prime}\right) .
$$

Insertion of the proposed distribution and the transition rates into this equation gives that $\alpha$ must be determined from

$$
\sum_{\{m, g: m+g=n\}} \psi(m) \theta(\boldsymbol{g}) H(\boldsymbol{m}+\boldsymbol{g})\left\{\sum_{\boldsymbol{g}^{\prime} \in G} \frac{c\left(\boldsymbol{g}^{\prime}\right) p\left(\boldsymbol{g}^{\prime}, \boldsymbol{g}\right)}{c(\boldsymbol{g})}-1\right\}=\alpha \phi(n) H(\boldsymbol{n}) .
$$

Assumption 2.1 shows that $\alpha$ is independent of $\boldsymbol{n}$ if and only if $\mu(\boldsymbol{g}, \boldsymbol{n})$ satisfies (2.1), and that $\alpha=\alpha^{\prime}-1$, which completes the proof.

REMARK (Service probabilities). Assumption (2.1) on $\mu(\boldsymbol{g}, \boldsymbol{n})$ is generally not required in a continuous-time model to obtain the equilibrium distribution as a product over the places. This assumption is required to obtain the result of Theorem 3.3. This illustrates part of the difference between the stationary distribution and the quasistationary distribution. A further difference is that quasi-local-balance for continuoustime models cannot be defined by analogy with $(2.6)$.

\section{Acknowledgements}

The research of R.J. Boucherie has been made possible by a fellowship of the Royal Netherlands Academy of Arts and Sciences. This research was carried out while the author was visiting the University of Adelaide supported by Australian 
Research Council Grant A69112151. Discussions with N.G. Bean and P.G. Taylor helped to improve the final result.

\section{References}

[1] R. J. Boucherie and N. M. van Dijk, "Product-forms for queueing networks with state dependent multiple job transitions", Adv. Appl. Prob. 23 (1991) 152-187.

[2] J. N. Darroch and E. Seneta, "On quasi-stationary distributions in absorbing discrete-time finite Markov chains", J. Appl. Prob. 2 (1965) 88-100.

[3] J. N. Darroch and E. Seneta, "On quasi-stationary distributions in absorbing continuous-time finite Markov chains", J. Appl. Prob. 4 (1967) 192-196.

[4] W. Henderson and P. G. Taylor, "Product-forms in networks of queues with batch arrival and batch services", Queueing Systems 6 (1990) 71-88.

[5] H. Kesten, "A ratio limit theorem for (sub) Markov chains on $\{1,2, \ldots\}$ with bounded jumps", Adv. Appl. Prob. 27 (1995) 652-691.

[6] E. Seneta, Non-negative matrices and Markov chains (Springer-Verlag, New-York, 1981).

[7] E. Seneta and D. Vere-Jones, "On quasi-stationary distributions in discrete-time Markov chains with a denumerable infinity of states", J. Appl. Prob. 3 (1966) 403-434.

[8] D. Vere-Jones, "Geometric ergodicity in denumerable Markov chains", Quart. J. Math. Oxford 13 (1962) 7-28. 